\title{
Influence of Wavelength on Glass Welding by Ultra-Short Laser Pulses
}

\author{
Kristian Cvecek $^{1,3}$, Florian Stenglein ${ }^{1}$, Isamu Miyamoto ${ }^{3,4}$ and Michael Schmidt ${ }^{1,2,3}$ \\ ${ }^{1}$ blz - Bayerisches Laserzentrum, Konrad-Zuse Str. 2-6, 91052, Erlangen, Germany \\ E-mail: k.cvecek@blz.org \\ ${ }^{2}$ Institute of Photonic Technologies, Friedrich-Alexander-University of Erlangen-Nuremberg, \\ Konrad-Zuse-Str. 3/5, 91052 Erlangen, Germany \\ ${ }^{3}$ SAOT - Erlangen Graduate School in Advanced Optical Technologies , Friedrich-Alexander- \\ University of Erlangen-Nuremberg, Paul-Gordan-Str. 6, 91052 Erlangen, Germany \\ ${ }^{4}$ Osaka University, Japan, Osaka University, 2-1 Yamada-Oka, Osaka 565-0871, Japan
}

\begin{abstract}
Glass welding by ultra-short pulsed laser pulses is a complex and highly dynamic process that is influenced by many parameters such as pulse repetition rate, feed speed, pulse duration or laser wavelength. Despite the complexity the understanding of the underlying processes has progressed far enough to support first industrial applications. However, the understanding of the underlying processes is still far from complete. Thus, in the present work we strive to close the knowledge gap regarding the wavelength influence of the ultra-short pulsed laser on the welding. We present an experimental comparison of glass welding by ultra-short pulses at $532 \mathrm{~nm}$ and $1064 \mathrm{~nm}$ for a range of different irradiation parameters such as pulse energy, pulse repetition rate and feed speed at a fullwidth-at-half pulse duration of 12 ps for D263 borosilicate glass. The results show smaller crack tendency for the $1064 \mathrm{~nm}$ wavelength.
\end{abstract}

DOI: 10.2961/jlmn.2017.02.0012

Keywords: laser materials processing, glass and other amorphous materials, ultra-fast material modification, glass welding, wavelength influence

\section{Introduction}

The development of glass welding technology by ultrashort pulsed (USP) lasers has progressed considerably from the first description by Tamaki in 2006 [1] inasmuch that first industrial applications already rely on it, e.g. [2]. Albeit the understanding of the underlying effects of this complex and highly dynamic process is steadily improved by experiments end modelling, e.g. see [3] and references therein, it is yet still far from complete. This is especially true for process parameters such as the laser wavelength or pulse duration, since comparative experiments require in such case higher technological effort than is typically necessary if parameters such as feed speed or pulse repetition are only to be studied. Since it seems reasonable that further understanding of the heat accumulation regime in USP laser based processing of glass might improve process applications we investigate in the present work the wavelength influence of the USP laser on the welding. Especially, we evaluate the suitability or, in other words, the benefit of using a certain wavelength for USP glass welding.

The structure of this paper is as follows. The experimental setup is described in section 2, the methods used for evaluation of the wavelength suitability are given in section 3 and the results are presented in section 4 with the discussion in section 5 .

\section{Experiment}

The experimental setup is shown schematically in Fig. 1. The laser provides pulses with 12 ps full-width-athalf (FWHM) pulse duration at an adjustable pulse repetition rate. The laser can be operated at wavelengths of 1064 $\mathrm{nm}$ and $532 \mathrm{~nm}$. In each case the laser beam is focused into the sample by an aspheric lens, designed explicitly for the wavelength. The NA of the aspheric lenses was 0.52 for $532 \mathrm{~nm}$ and 0.51 for $1064 \mathrm{~nm}$. Prior to passing the aspheric lens the laser beam was expanded and collimated by a Galileo-telescope-like lens setup. Since the concave lens used in this telescope was the same for both wavelength experiments, it was necessary to optimize its distance to the collimating lens in order to provide a collimated beam in front of the aspheric lenses independently on the change of refractive power due to the wavelength dependency of the refractive index. The optimization was carried out by generating plasma in air by the aspheric lens and maximizing its brightness (of the plasma) for a given laser pulse energy (laser power) by adjusting the distance inside the telescope.

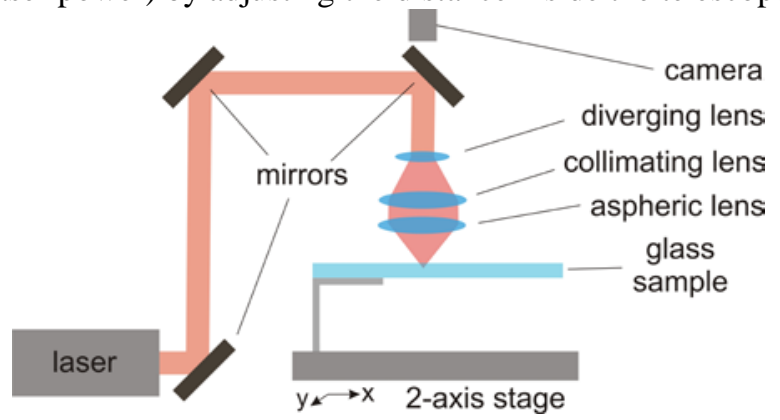

Fig. 1 Schematics of the experimental setup.

Please note: since the NAs of the aspheric lenses are very similar, the diameter of the focal spot will be half as big for $532 \mathrm{~nm}$ than for $1064 \mathrm{~nm}$ in an ideal case resulting in a 4 times higher intensity inside the focal spot for 532 $\mathrm{nm}$. However, as has been shown in several publications, e.g. $[3,4,5]$ the best suited position for welding is not the geometrical focus, but - as the molten zone widens toward 
the laser beam due to an interplay between heat accumulation and avalanche ionization - several tens of microns above the nominal focus position. At this position, the shape of the welding seam that is important for the joining is not determined by the focal spot size but by the beam divergence [7], compare also cross sections in Fig. 6. Thus, to provide application relevant results we decided to compare beams at different wavelengths with similar beam divergence instead of similar focal size. The ramifications of this choice are further discussed in section 5.

The irradiated samples consisted of $1 \mathrm{~mm}$ thick D263 glass plates. The samples were mounted on an $x-y-$ translational stage and were aligned horizontally first by confocally measuring the distance from the aspheric lens to the upper surface of the sample for several $x$-y-positions. The measurement results were used to calculate the tilt angle necessary for a horizontal alignment. After horizontal alignment the focal spot was displaced geometrically by $133 \mu \mathrm{m}$ into the sample resulting in a nominal focus position of approx. $200 \mu \mathrm{m}$ below the upper surface due to the refractive index of roughly $\sim 1.5$ for the D263 glass.

During the experiments the processing parameters, that is the pulse energy, pulse repetition rate and feed speed were varied for both wavelengths. The pulse energy was varied in 20 steps by setting the average laser power from 0 to approximately $3 \mathrm{~W}$ for all pulse repetition rates thereby achieving maximum pulse energies from 1.5 to $15 \mu \mathrm{J}$ (depending on the pulse repetition rate). The values for the investigated feed speeds and pulse repetition rates are given in Table 1.

Table 1 Investigated process parameters

\begin{tabular}{cc}
\hline Parameter & Values \\
\hline Wavelength in $\mathrm{nm}$ & $532 / 1064$ \\
Feed speed in $\mathrm{mm} / \mathrm{s}$ & $20 / 50 / 100$ \\
$\begin{array}{c}\text { Pulse repetition rate } \\
\text { in } \mathrm{MHz}\end{array}$ & $0.2 / 0.5 / 1 / 2$ \\
\hline
\end{tabular}

\section{Methods}

In this section we formulate the criteria that allow us to evaluate which wavelength is better suited for USP based glass welding. Not every possible criterion is suitable, though: while e.g. the ionization threshold would provide a stronger absorption for the shorter wavelength in the single pulse regime (due to a relaxation of the number photons necessary to drive the multi-photon ionization process), it is not clear whether this fact holds also in the heat accumulation regime that might or might not be strongly driven by avalanche ionization and Inverse Bremsstrahlung.

Therefore, we formulate criteria that are based on the typical necessities of the applications (e.g. glass MEMS for medical applications) USP glass welding process such as high load capacity of the welding seams and high hermeticity. Further criteria e.g. processing speed or cost effectiveness could also be formulated, but the above mentioned criteria are the most important for a robust process.

Both criteria are closely related to the joining area of the welding seam without cracks, assuming a more or less constant welding seam strength and negligible internal pressure inside the welding seam. The simplification introduced by the last two conditions needs to be kept in mind. This is because during USP processing of glass in the heat accumulation regime the glass experiences an irreversible volume expansion (see $[8,9]$ ) that provides an internal pressure to the welding seam. For very large welding seams this pressure can cause the surrounding glass to break as shown in Fig. 2. However, if the welding seam crosssectional area is sufficiently small the pressure will not damage the glass.
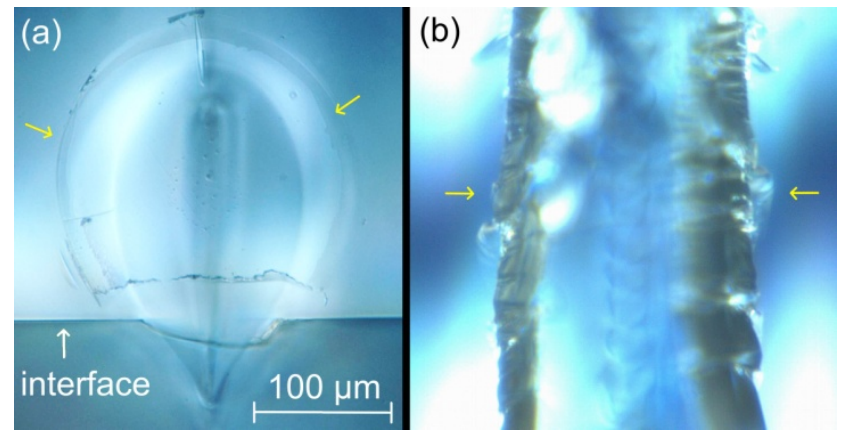

Fig. 2 Cross section (a) and top view (b) of a large welding seam in D263 glass causing cracks indicated by yellow arrows. (Conditions: $1064 \mathrm{~nm}$, pulse energy $8 \mu \mathrm{J}$, feed speed $20 \mathrm{~mm} / \mathrm{s}$, repetition rate $1 \mathrm{MHz}$. (taken from [5] at an NA of 0.55)

It is intuitively clear that a bigger molten zone is partly a result of higher temperatures during the process, giving the melt a lower viscosity and higher molecule diffusivity resulting in a better mixing of the joining partners. This can be observed in Fig. 2 where besides the cracks, a defect is visible at the interface of the glass showing an "indented" surface. Obviously the temperature is not high enough to completely overcome the surface tension of the interface. This is corroborated by simulations in e.g. [10] that predict a temperature decrease towards the tip. However, the observed indentation in Fig. 2 is not always present. Even so, the imperfect joining between the glass plates can be shown by etching using the (acidic) Sirtl-solution [11]. Polished cross sections of welding seams, welded in optical contact similar to [13] and post-processed with this etching method are shown in Fig. 3.

Since the preparation of optical contact constitutes the preparation of a gap smaller than at least $100 \mathrm{~nm}$, the black line visible in Fig. 3 as a kind of gap has been caused by the etching process - since the glass is not chemically bonded, e.i. welded, the etchant can ingress into the area of the optical contact (due to its small molecule size) and corrode the glass surfaces.

Following this realization we actually do not need to prepare polished and etched cross sections of welded and optically pre-contacted glass samples as in [13] or Fig. 3 for 240 melt runs (given by the number of all investigated parameters). Instead, it is sufficient to observe the width of the molten zone of a melt-line in a single glass plate from the top, Fig. 4. This is possible, because the cross sections shapes for both wavelengths are very similar, see Fig. 6. A further advantage is the possibility to observe cracks at the same time. (The evaluation of cracks will be addressed later in this section.) 

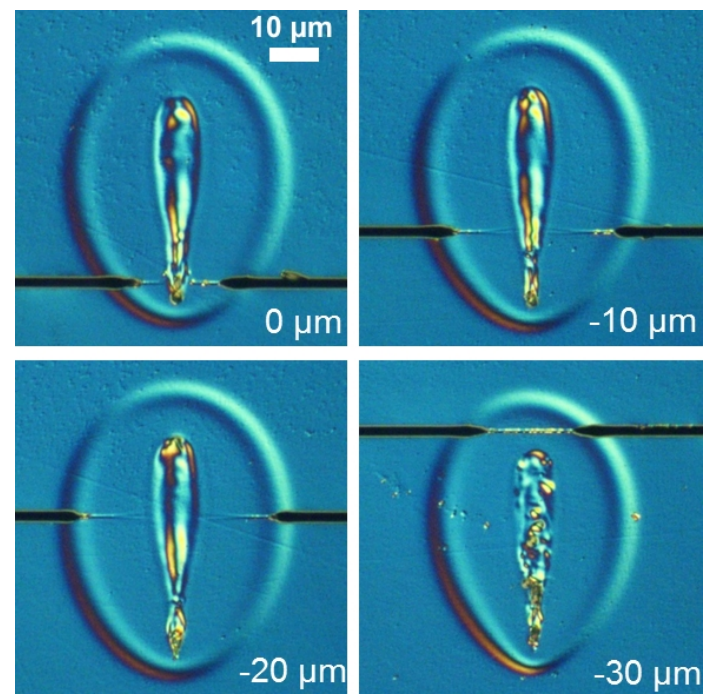

Fig. 3 Sirtl-etched cross sections of welding seams at different displacements of the focal spot with respect to the joining interface. The laser beam impinges from the top. (1064 nm, 20 mm/s, 1 MHz, $1.2 \mu \mathrm{J}$, NA 0.55)

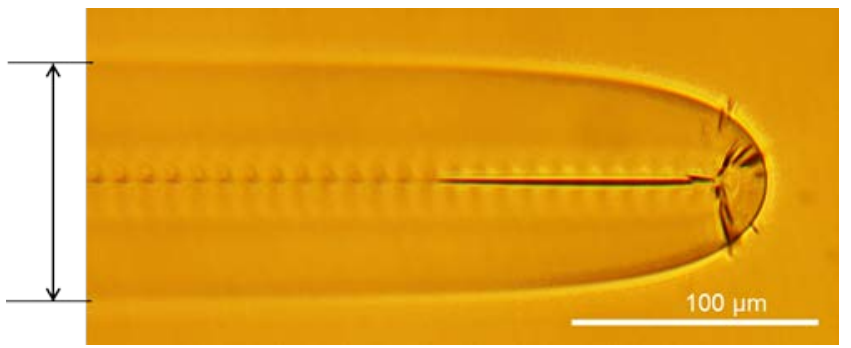

Fig. 4 Top view on a melt line in glass, making the maximum achievable width as well as any cracks well visible. (532 nm, $20 \mathrm{~mm} / \mathrm{s}, 0.2 \mathrm{MHz}, 12.1 \mu \mathrm{J}$, NA $0.52)$

The maximum achieved width of the welding seam is determined for a given combination of pulse repetition rate and feed speed for both wavelengths depending on the laser pulse energy. An example of this is shown for a feed speed of $20 \mathrm{~mm} / \mathrm{s}$ and $0.2 \mathrm{MHz}$ pulse repetition rate in Fig. 5. It is obvious that $532 \mathrm{~nm}$ provides a larger molten zone and, thus, must experience a higher absorption.

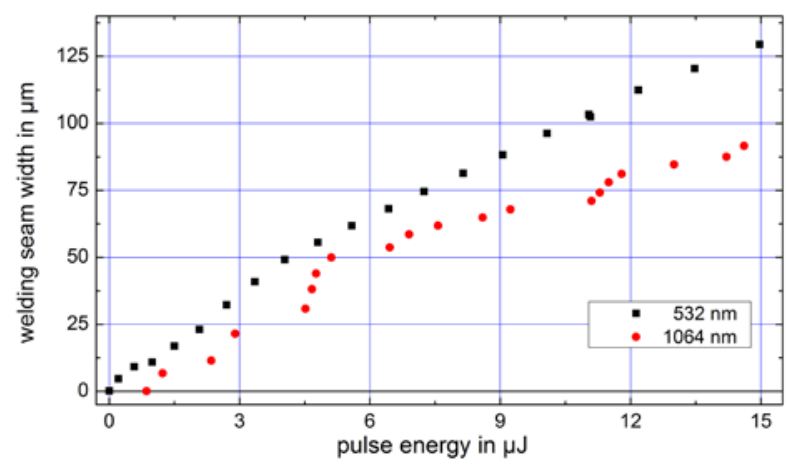

Fig. 5 Comparison of the pulse energy dependent maximum seam width for both wavelengths at a feed speed of $20 \mathrm{~mm} / \mathrm{s}$ and $0.2 \mathrm{MHz}$ pulse repetition rate.

With this it is fairly easy to determine the improvement in melt run width that is achieved e.g. by using low order polynomial functions to fit the data. This way the seam width as well as the difference between the seam width can be calculated for any given pulse energy or seam width in question. Especially the pulse energy difference can be considered as a kind of width improvement using the shorter wavelength in this case. This was exemplary done for the data in Fig. 5 for a welding seam width of $50 \mu \mathrm{m}$ resulting in a width improvement of $1.5 \mu \mathrm{J}$ for $532 \mathrm{~nm}$ over the $1064 \mathrm{~nm}$ and is shown in Fig. 7. Of course, using the fitted functions we can assign WI to every seam width of interest that was obtained in the experiment - outside the observed width range the fitted function will not provide reliable values.
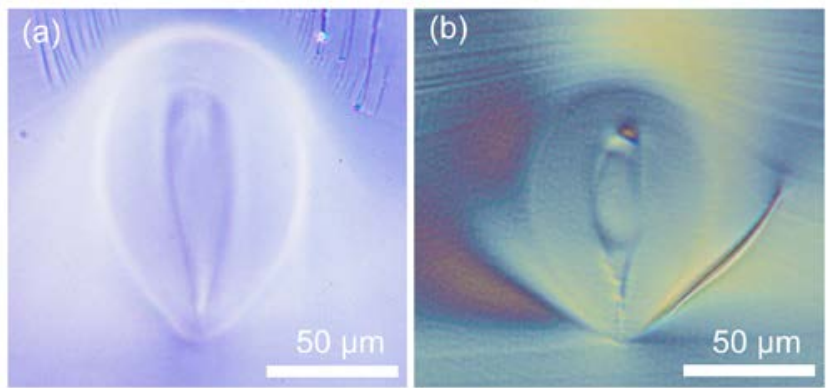

Fig. 6 Comparison of cross-sections for (a) $532 \mathrm{~nm}$ at NA0.52 and (b) $1064 \mathrm{~nm}$ at NA 0.51, both at a feed speed of $20 \mathrm{~mm} / \mathrm{s}$.

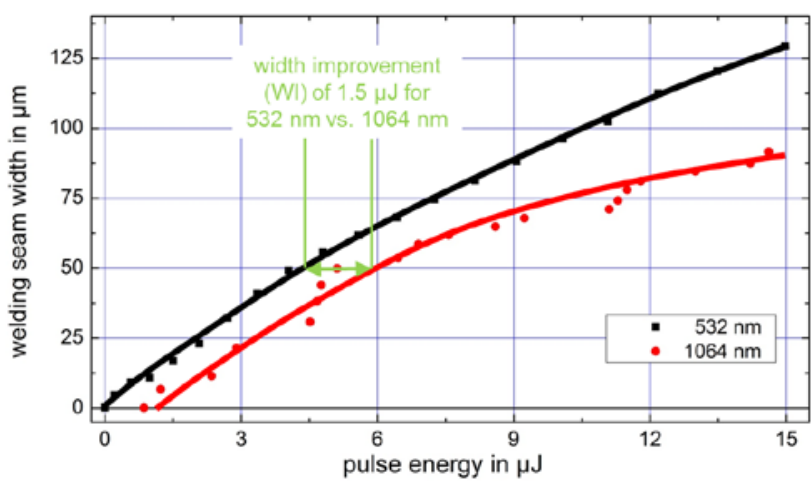

Fig. 7 Schematic explaining the method to determine the width improvement for a width of $50 \mu \mathrm{m}$.

The cracks are evaluated as follows: depending on the applied pulse energy the melt lines (see Fig. 4) are classified whether they are crack-free, exhibit a single crack or do have two or more cracks. The effects of both wavelengths are compared in a diagram, as shown in Fig. 8 for the same experimental conditions that were used in Fig. 5 and 7.

While we could in principle define similarly to the above mentioned width improvement also a kind of crack improvement, see Fig. 8, this is not helpful, because for larger pulse energies which cracks will always be present. Instead, since already a single crack might strongly reduce the hermeticity and as the occurrence of cracks is spurious, we define a crack threshold energy (CTE) as the average of the pulse energy at which the first single crack has been observed and the maximum pulse energy at which and below which no cracks have been found (Fig. 8). In order to compare the achievable widths at both wavelengths the corresponding CTEs need to be taken into account. The data for the width improvement and the CTEs can now be used to determine if a certain seam width can be achieved without cracks. An example for this is shown in Fig. 9 for a 
seam width of $50 \mu \mathrm{m}$. Because this kind of evaluation can be conducted for any seam width it is possible to determine for every investigated experimental condition the maximum achievable crack-free seam width. This width can be readily plotted as height in a contour plot diagram for each wavelength, and is shown in Fig. 11.

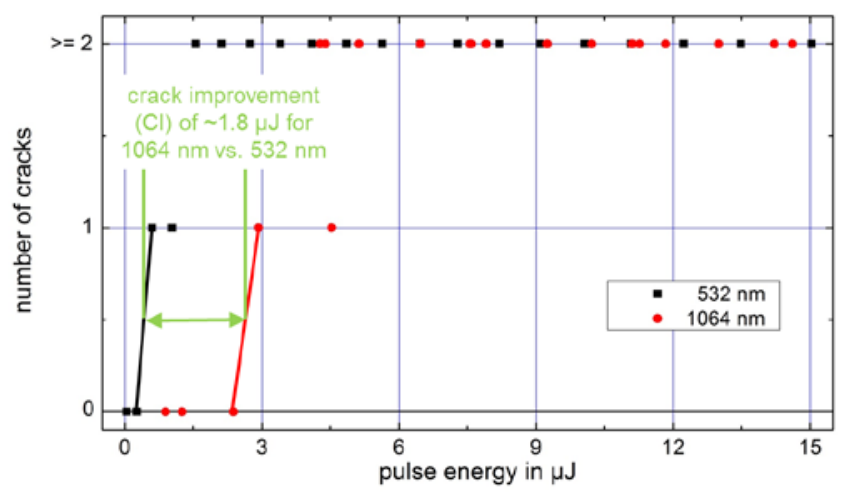

Fig. 8 Schematic explaining the method to determine the crack threshold energies $(20 \mathrm{~mm} / \mathrm{s}, 0.2 \mathrm{MHz})$.

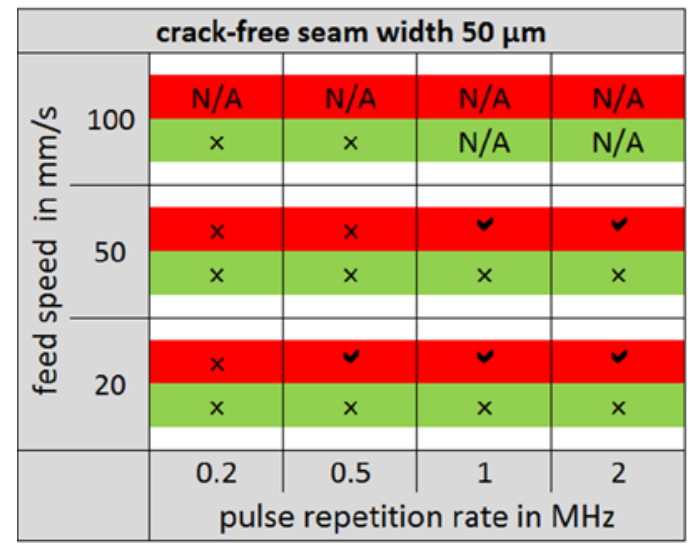

Fig. 9 Exemplary evaluation of the experimental results for crack-free seam width of $50 \mu \mathrm{m}$. Red and green rows depict the results for $1064 \mathrm{~nm}$ and $532 \mathrm{~nm}$, respectively. The symbols “ $\checkmark$ ”, “×”, “N/A” stand for the cases when the seam was achieved without cracks, achieved but with one or more cracks or not at all, respectively.

\section{Results}

The evaluation of the width improvements shows for all investigated processing conditions a larger seam width for $532 \mathrm{~nm}$ than for $1064 \mathrm{~nm}$, of at least $0.4 \mu \mathrm{J}$ for $532 \mathrm{~nm}$. Depending on parameters and the seam width in question the improvement can become as large as $6 \mu \mathrm{J}$ (observed for a seam width of $80 \mu \mathrm{m}$ at $0.2 \mathrm{MHz}$ and $20 \mathrm{~mm} / \mathrm{s}$ ). Please note that this value is given by the extents of the maximum investigated pulse energies $(15 \mu \mathrm{J})$ in the experiment. The results show a tendency to expect even bigger width improvements at $532 \mathrm{~nm}$ for higher pulse energies.

The CTE in Fig. 10 shows that it is always lower for $532 \mathrm{~nm}$ than for $1064 \mathrm{~nm}$, e.i. $532 \mathrm{~nm}$ irradiation causes even at lower pulse energies cracks. As is shown by Fig. 11, this cannot even be offset by the larger seam width achieved at $532 \mathrm{~nm}$. Furthermore, in the course of our experiments, using high pulse repetition rates of 1 and $2 \mathrm{MHz}$ at $1064 \mathrm{~nm}$ has allowed to completely avoid the formation of cracks.

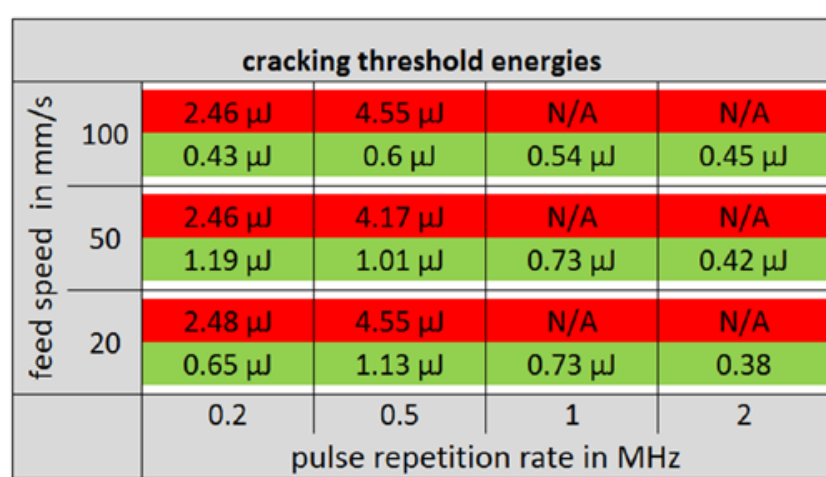

Fig. 10 Cracking threshold energies (CTEs) for $1064 \mathrm{~nm}$ (red rows) and $532 \mathrm{~nm}$ (green rows). "N/A" means that no cracking was observed.

The maximum achievable crack-free seam width is shown for both wavelengths in Fig. 11. (Please note, the contour lines are linearly interpolated between the measured parameter sets of pulse repetition rate and feed speed - compare Table 1). As can be seen the maximum achievable crack-free seam width is larger for all investigated parameters (pulse repetition rate, feed speed as well as pulse energy). The largest achieved maximum crack-free seam width is $70 \mu \mathrm{m}$ for $1064 \mathrm{~nm}$ and $30 \mu \mathrm{m}$ for $532 \mathrm{~nm}$.

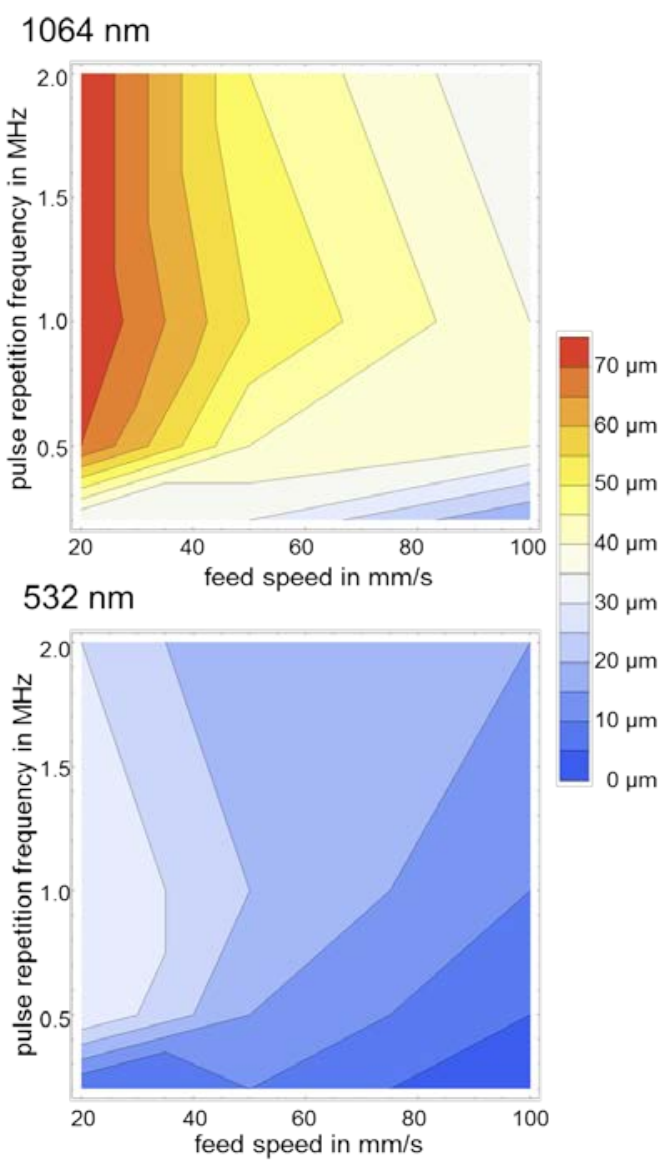

Fig. 11 Maximum achievable crack-free seam width for $1064 \mathrm{~nm}$ (upper diagram) and $532 \mathrm{~nm}$ (lower diagram).

\section{Discussion}

Obviously, following Fig. 11 glass welding by ultrashort laser pulses at 10 ps FWHM pulse duration provides a more reliable process (regarding the achievement of broad 
welding seams without cracks) using $1064 \mathrm{~nm}$ than $532 \mathrm{~nm}$. This is observed in spite of the fact that $532 \mathrm{~nm}$ provides in all cases wider welding seams which means that $532 \mathrm{~nm}$ exhibits a significantly higher absorptivity than $1064 \mathrm{~nm}$ not during the initial ionization stage by multiphoton ionization but also during the heat accumulation regime.

However, the cracks were predominantly observed at the beginning of the seam - as in the tip of the melt line in Fig. 4. This, in turn, can be interpreted as crack formation at a stage when the glass is relatively cold and brittle and the heat accumulation regime has not yet achieved its "quasi" steady state with a low viscosity melt. Otherwise, the cracks would not be observed in the middle of the molten zone but at its edges, compare $[5,12]$ and would show a tunnel-like shape as in Fig. 2 (a). The origin of such cracks can be attributed to the internal stresses of the welding seam, caused either by irreversible volume expansion [8]

In consequence, the cracks observed in e.g. Fig. 4 must have formed during the irradiation of the glass with the first impingent pulses. Afterwards, with increasing heat accumulation the material will melt, and, if the growth rate of the melt front is faster than the feed speed, the melt will close the cracks formed at the beginning - unless the cracks are too large from the beginning or the melt front propagation speed is too low. Therefore, the crack formation problem during glass welding can be idealized for small seam areas by considering single pulse interaction with the cold glass.

In this case, the second harmonic will interact much stronger with the material assuming similar pulse energy and duration than a pulse at a longer wavelength, because as has been already pointed out in section 2 - the intensity inside the focus spot is roughly 4 times larger and the multi-photon-ionization threshold is significantly lower. This is corroborated by experiments and simulations presented in $[14,15]$

Of course, this means that using the shorter wavelength provides a process that is much more energy efficient than one at a longer wavelength. Of course, a reduction of the NA of the focusing optics would result in lower focal spot intensities and thus provide a more gentle interaction between the pulses and the material, but it would also result in a decrease of the beam divergence which would be not suitable for all cases or applications. Especially, if the tear drop shaped molten zone is required to have a certain width but limited height as e.g. in [8], the aperture angle of the focused beam is an experimental condition that must be kept constant.

One possible way to reduce the intensity of the focal spot while preserving the aperture angle would be to use a laser beam which has a beam-parameter-product that is larger than the ideal Gaussian beam. This, way the beam divergence could be kept similar to the experiments conducted here, but the focal spot would be significantly larger and, thus, possibly reduce the cracking problem. Very likely, adaptive optics such as a spatial light modulator could help to optimally tailor the beam properties to achieve a reduced focal spot diameter while preserving the beam divergence at the beginning of the welding seam. Ultimately, using this approach could also help to conduct glass welding at shorter wavelengths leading to improved energy efficiency or processing speed.

\section{Conclusion}

From the presented experimental results can be concluded that absorptivity of the shorter wavelength is higher for during the whole process. While in the heat accumulation regime this causes a significantly larger molten zone, the higher absorptivity is detrimental at the start of the process, because it causes cracks at lower pulse energies than the longer wavelength. Consequently, without active optics for beam shaping, larger maximum width of a crack-free welding seam is achieved by the longer wavelength. However, since the efficiency in terms of energy and process speed is higher for the shorter wavelength it could be worthwhile for high throughput industrial applications of USP glass welding to investigate the effect of shorter wavelengths in combination with active beam shaping.

\section{References}

[1] T. Tamaki, W. Watanabe, and K. Itoh: Opt. Express, 14, (2006) 10460.

[2] E. Kaiser, A. Killi, A. Budnicki, S. Pricking and M. Kumkar: Physics Procedia, 83, (2016).

[3] I. Miyamoto, Y. Okamoto, R. Tanabe, Y. Ito, K. Cvecek and M. Schmidt: Opt. Express, 24, (2016) 25718.

[4] K. Cvecek, I. Miyamoto, J. Strauss, V. Bui, S. Scharfenberg, T. Frick and M. Schmidt: JLMN-Journal of Laser Micro/Nanoengineering, 7, (2012) 68.

[5] K. Cvecek, I. Miyamoto, I. Alexeev and M. Schmidt: Physics Procedia, 5, (2010) 495.

[6] K. Cvecek, I. Miyamoto, M. Adam and M. Schmidt: Physics Procedia, 39, (2012) 563.

[7] P. Kongsuwan and Y. L. Yeo: Proc. ICALEO, Anaheim, (2010).

[8] K. Cvecek, R. Odato, S. Dehmel, I. Miyamoto, and M. Schmidt: Opt. Express 23, (2015) 5681.

[9] Y. Bellouard: Proc. of Laser Precision Microfabrication, Vilnius, (2014).

[10] I. Miyamoto, K. Cvecek, Y. Okamoto and M. Schmidt: Appl. Phys. A, 11, (2013) 187.

[11]A. F. Bogenschütz: „Ätzpraxis für Halbleiter“, (Carl Hanser Verlag, Munich, 1967), p. 96.

[12]E. Sharon and J. Fineberg: Adv. Eng. Mater., 1, (1999) 119.

[13] K. Cvecek, I. Miyamoto, J. Strauss, M. Wolf, T. Frick, and M. Schmidt: Appl. Opt., 50, (2011) 1941.

[14] I. Miyamoto, K. Cvecek and M. Schmidt: Proc. Of Proc. of Laser Precision Microfabrication, Toyama, (2017).

[15] M. D. Perry, B. C. Stuart, P. S. Banks, M. D. Feit, V. Yanovsky, and A. M. Rubenchik: J. Appl. Phys., 85, (1999) 6803. 